Relato de Experiência

Revista Saúde.Com

Rev. Saúde.Com 2020; 16(1): 1766-1773

ISSN 1809-0761

www.uesb.br/revista/rsc/ojs

\title{
ESTÁGIO EM SAÚDE COLETIVA COMO ESTRATÉGIA DE REORIENTAÇÃO DA FORMAÇÃO EM ODONTOLOGIA: UM RELATO DE EXPERIÊNCIA
}

\author{
PUBLIC HEALTH INTERNSHIP AS A REORIENTATION STRATEGY FOR TRAINING IN \\ DENTISTRY: A REPORT OF EXPERIENCE
Marcela Beatriz Aguiar Moreira, Marília de Matos Amorim, Cristiane Brandão Santos
Almeida, Alessandra Laís Pinho Valente Pires \\ Universidade Estadual de Feira de Santana - UEFS
}

\begin{abstract}
This article aims to report the role of the curricular component Internship in Public Health I, as a strategy of reorientation in dentistry training, for students of a private college in the municipality of Feira de Santana, Bahia. This is a descriptive and exploratory experience report of teaching-service integration of the Dentistry Course of the Higher Education Unit of Feira de Santana (UNEF) in Family Health Units (FHU) of Feira de Santana, Bahia. The Internship was developed during the first semester of 2018, with about 100 students from the four semester of the referred course. The students were allocated to twelve FHU, in which they developed actions, including home visits, educational activities for the community and health team, as well as actions in the school environment, including supervised brushing and oral health epidemiological surveys. Thus, this discipline enabled the academic participation in the development of health education actions, with the exchange of theoretical and practical knowledge about oral and general health, besides enabling the student to live the reality of the Unified Health System (SUS), sensitizing them to the social practice inherent in working in this system. Therefore, this internship stimulates the student's commitment to their role as citizens, students and future health professionals, based on the principles and guidelines of the SUS.
\end{abstract}

Keywords: Dentistry, Public Health, Unified Health System.

\section{Resumo}

$O$ presente artigo tem como objetivo relatar o papel do componente curricular Estágio em Saúde Coletiva I, como estratégia de reorientação na formação em odontologia, para discentes de uma faculdade privada do município de Feira de Santana, Bahia. Trata-se de um relato de experiência de caráter descritivo e exploratório, de integração ensino-serviço do Curso de Odontologia da Unidade de Ensino Superior de Feira de Santana (UNEF) em Unidades de Saúde da Família (USF) de Feira de Santana, Bahia. O Estágio foi desenvolvido durante o primeiro semestre de 2018, com cerca de 100 graduandos do quarto semestre do referido curso. Os acadêmicos foram alocados em doze USF, nas quais desenvolveram ações, incluindo visitas domiciliares, atividades educativas para a comunidade e equipe de saúde, bem como ações em ambiente escolar, incluindo escovação supervisionada e levantamentos epidemiológicos em saúde bucal. Destarte, essa disciplina possibilitou a participação acadêmica no desenvolvimento de ações de educação em saúde, com trocas de conhecimentos teórico-práticos sobre a saúde bucal e geral, além de oportunizar ao discente viver a realidade do Sistema Único de Saúde (SUS), sensibilizando-o a prática social inerente ao trabalho neste sistema. O referido estágio estimula, portanto, o compromisso do discente perante seu papel como cidadão, estudante e futuro profissional de saúde, pautados nos princípios e diretrizes do SUS.

Palavras-chave: Odontologia, Saúde Coletiva, Sistema Único de Saúde. 
assim,preparando-os para o mercado de trabalho e para as demandas sociais ${ }^{6,2}$.

A consolidação do Sistema Único de Saúde (SUS), em consonância à Reforma Sanitária, e às conquistas pelos direitos à saúde são consideradas exemplos de propostas democráticas que ocasionaram, e vem promovendo, avanços no setor saúde do Brasil ${ }^{1}$. Para tanto, é sabido que o papel dos profissionais de saúde que fomentam esse sistema é de fundamental relevância para a concretização efetiva dos princípios e diretrizes do SUS. E por isso, torna-se basilar a orientação/reorientação na formação desses atores em saúde, a fim de edificar um perfil profissional comprometido com uma atuação de qualidade, eficiência e resolubilidade no sistema de saúde do país ${ }^{2,3}$.

Neste sentido, dando ênfase a Odontologia, o ministério da Educação (MEC) publicou as Diretrizes Curriculares Nacionais (DCN) na Resolução CNE/CES № $3^{3}$, de 19/02/2002, as quais apontam elementos conceituais, filosóficos e metodológicos que possam fundamentar a mudança no processo de educação em Odontologia. Essas diretrizes são baseadas no desenvolvimento de competências e habilidades requeridas por todos os profissionais da saúde, e apontam novas configurações para os padrões curriculares até então vigentes, indicando a necessidade de uma reestruturação dos cursos de graduação, com mudanças paradigmáticas no contexto acadêmico, a exemplo da inserção de atividades de estágio em saúde, em diferentes cenários de práticas, realizadas em contato com a realidade social.

É notório que, ainda assim, a formação odontológica tem sua prática pautada no modelo flexneriano, e os profissionais graduados sob a

influência deste padrão apresentam características com vistas a odontologia curativa, mecanicista e aptos para atuar no sistema privado, sem priorizar as necessidades da saúde pública $^{4,5}$. Entretanto, com a inserção do acadêmico na vivência do SUS, tem sido possível alçar mudanças desse paradigma, ou seja, formar indivíduos mais comprometidos com a coletividade, capacitando-os a ter uma percepção mais abrangente, dinâmica,

complementar e integrada, com desenvolvimento crítico e reflexivo, dotados de conhecimentos que os habilitem a uma prática competente, ética e socialmente responsável, e

Dessa forma, salienta-se que é essencial para a formação do cirurgião-dentista a vivência em situações reais do cotidiano do sistema de saúde brasileiro. Com a possibilidade de ir a campo e fazer parte do serviço, estimula-se o desenvolvimento do trabalho em equipe, a interdisciplinaridade, a qualificação de recursos humanos destinados ao sistema de saúde, o cuidado integral à saúde dos usuários e a melhoria da qualidade de vida da população ${ }^{7}$.

Contextualizando o exposto com a temática desse estudo, destaca-se que o curso de Odontologia da Unidade de Ensino Superior de Feira de Santana (UNEF) tem por objetivo construir um perfil do cirurgião-dentista com visão ampla da saúde e realidade social, preparados para o mercado de trabalho, sobretudo, no ponto de vista técnico, científico, humano e ético, com enfoque em promover saúde bucal da população. Com destaque ao componente curricular Estágio em Saúde Coletiva I, do curso de Odontologia da UNEF, este visa inserir o estudante de odontologia na atenção básica com abordagem na prevenção de doenças e agravos, e na promoção da saúde. $E$ assim, juntamente com as demais disciplinas ao decorrer do curso, torna-se possível formar profissionais generalistas, humanistas, com visão crítica e reflexiva, para atuar em todos os níveis de atenção à saúde com base no rigor técnico e científico.

Neste sentido, o objetivo deste artigo é relatar o papel do componente curricular Estágio em Saúde Coletiva I, como estratégia de reorientação na formação em odontologia, para discentes de uma faculdade privada do município de Feira de Santana, Bahia.

\section{Relato de Experiência}

Trata-se de um relato de experiência de caráter descritivo e exploratório, de integração ensino-serviço do Curso de Odontologia da Unidade de Ensino Superior de Feira de Santana (UNEF) em Unidade de Saúde da Família (USF) na cidade de Feira de Santana, Bahia.

Este relato foi desenvolvido pela equipe docente da disciplina "Estágio em Saúde Coletiva I", desenvolvida durante o primeiro semestre do ano de 2018. Este componente faz parte da 
matriz curricular de 80 horas, do quarto período do curso de odontologia - UNEF, e contou com cerca de 100 graduandos.

A UNEF é uma instituição de ensino privada de referência localizada no município de Feira de Santana, na Bahia. O município está localizado cerca de 108 km da capital do estado, Salvador, é considerada a segunda maior cidade do Estado da Bahia/Brasil, considerada um dos mais importantes entroncamentos rodoviários do Norte e Nordeste do Brasil. De acordo com os dados do Instituto Brasileiro de Geografia e Estatística (IBGE), este município possui uma população, estimada para o ano de 2018 , de 609.913 habitantes, além de área territorial $1.304,425 \mathrm{~km}^{2}$, e densidade demográfica de $416,03 \mathrm{hab} / \mathrm{km}^{2} 8$.

No início do semestre, os acadêmicos foram divididos em grupos, sendo alocados aleatoriamente para uma USF do município. As Unidades de saúde foram escolhidas mediante definição da Prefeitura de Feira de Santana, sendo contempladas: USF Tomba I e III, USF Gabriela III e IV, USF Feira X, USF Corredor dos Araçás, USF Parque Getúlio Vargas I e II, USF Sobradinho I e II, USF Aviário III e IV, USF Alto do Papagaio I e II, USF Eucaliptos, USF Santo Antônio dos Prazeres e USF Santa Monica II. Das doze USFs, apenas três tinham a Equipe de Saúde Bucal agregada a Equipes de Saúde da Família, como a USF Gabriela III e IV, Parque Getúlio Vargas I e II, e Alto do Papagaio I e II. Dessa forma, o local de trabalho foi à própria sede da USF, bem como escolas, creches, praças públicas, empresas privadas, e locais disponibilizados na comunidade.

As atividades aconteceram de modo programado com as equipes durante uma semana de reunião na unidade de saúde. Com planejamento conjunto da equipe de saúde e equipe acadêmica, mediada pelo docente, foi definida uma programação de quatro meses. Planejou-se, então, ações semanais, incluindo visitas domiciliares, atividades educativas para a comunidade (oficinas, rodas de conversas, palestras, salas de espera, capacitação e feira de saúde), envolvendo grupos específicos (ações integradas no Hiperdia, Pré-natal, Puericultura, Saúde da mulher, Saúde do homem e Saúde do idoso), bem como foram planejados ações em ambiente escolar, incluindo escovação supervisionada e levantamentos epidemiológicos em saúde bucal, a fim de conhecer as necessidades de saúde bucal da comunidade assistida.

Assim, seguindo o planejamento conjunto, foram realizadas visitas domiciliares nas comunidades, onde houve diálogos com as famílias assistidas, instruções de saúde bucal, esclarecimento de dúvidas e possíveis caminhos de acesso ao cirurgião-dentista nas unidades que não contavam com esse profissional. A fim de guiar as observações, utilizou-se 0 instrumento de coleta de dados Ficha SB Brasil 2010, o qual foi utilizado em pesquisas nacionais de saúde bucal, conduzidas pelo Ministério da Saúde do Brasil, como o SB Brasil 2010․ Para a instrução sobre saúde bucal foram utilizados materiais didáticos como macromodelos, distribuições de panfletos educativos e distribuição de kits de higiene oral, os quais continham escovas dentais e creme dental.

Dentre as atividades desenvolvidas, a maioria foi realizada dentro do contexto de cada USF. De forma geral, foram executadas para grupos específicos, como idosos, gestantes, puérperas, mulheres, homens, equipe de saúde e demanda espontânea. Destacou-se a apresentação de oficinas, rodas de conversas, palestras e salas de espera sobre cárie, doença periodontal e câncer de boca, evidenciando a importância da higiene para saúde e como os citados produtos podem auxiliar na prevenção das doenças, além da apresentação das técnicas de escovação dental, sempre em busca da interação com o público, considerando o conhecimento prévio da população, e favorecendo, assim, o processo ensinoaprendizagem.

Também foram realizadas, em algumas unidades de saúde, capacitações sobre saúde bucal e doenças bucais para profissionais de saúde, tais como os agentes comunitários de saúde, técnicos de enfermagem e enfermeiros. É válido salientar que essas atividades também ocorreram em outros locais na comunidade, como no Centro de Referência a Assistência Social (CRAS), envolvendo grupo de idosos, em empresa privada, abrangendo grupo de homens, e em praças públicas, abarcando a comunidade em geral.

A fim de alcançar o público infantil, foram realizadas ações educativas em ambiente escolar. 0 público alvo variou entre 30 a 200 crianças por 
escola, e com faixa etária entre 03 a 15 anos. Repletas de ludicidade, encantos e criatividade, as atividades foram feitas com uma diversidade de materiais didáticos, incluindo: macromodelos, fantoches, dentes de isopor, cartazes e panfletos informativos. De acordo com a faixa etária, foi possível introduzir informações sobre saúde bucal, doenças bucais, como cárie e doença periodontal, doenças sexualmente transmissíveis, alimentação e hábitos saudáveis. E para possibilitar o aprendizado prático das técnicas de escovação, foi feita escovação supervisionada, com o suporte do escovódromo, disponibilizado pela UNEF, materiais de biossegurança e kits de escovação.

Com o intuito de avaliar a condição de saúde bucal dessas crianças e adolescentes assistidos nas atividades escolares, foram feitos levantamentos epidemiológicos em saúde bucal. Como suporte metodológico, foi utilizado o Manual do examinador do Projeto SB Brasil 2000, disponibilizado pelo Ministério da Saúde do Brasil - Área Técnica de Saúde Bucal ${ }^{10}$. Para a coleta dos dados, empregou-se um instrumento mais atualizado, a ficha de exame do Projeto SB Brasil $2010^{9}$, a qual foi utilizada em pesquisas nacionais de saúde bucal, conduzidas pelo Ministério da Saúde. É válido ressaltar que para essas atividades foram avaliados os Índices Cárie Dentária e Necessidade de Tratamento. A avaliação bucal foi feita por um examinador, que possuía o auxílio de um anotador, ambos previamente calibrados. Foram utilizados materiais de biossegurança para o exame bucal e materiais de suporte para a anotação dos dados.

Os dados coletados durante o estágio foram tabulados, analisados e apresentados às respectivas USFs, onde houve discussões entre equipe acadêmica e equipe de saúde sobre os resultados encontrados. Ademais, os relatórios finais de cada unidade foram encaminhados e apresentados a gestão municipal de saúde.

Assim, o relato foi baseado no desenvolvimento das estratégias executadas no referido Estágio em Saúde Coletiva, nas experiências adquiridas pela convivência com a equipe acadêmica, equipe de saúde e comunidade e nos desafios enfrentados ao longo deste processo.

\section{Resultados e discussões}

Ao permitir a aproximação dos acadêmicos com a estrutura, a dinâmica de funcionamento do sistema de saúde do município de Feira de Santana, através do Estágio em Saúde coletiva I, possibilitou a estes a troca de saberes entre diversos atores sociais, profissionais e usuários. Contribuindo assim, para a qualificação dos futuros profissionais nas perspectivas de reorientação da formação em odontologia, isto é, uma formação voltada ao fortalecimento do SUS assim como é preconizada pelas Diretrizes Curriculares Nacionais (DCN) dos Cursos de Odontologia na resolução CNE/CES 3, de 19 de fevereiro de $2002^{3}$ :

“Art. 3o o Curso de Graduação em Odontologia tem como perfil do formando egresso/profissional o Cirurgião Dentista, com formação generalista, humanista, crítica e reflexiva, para atuar em todos os níveis de atenção à saúde, com base no rigor técnico e científico. Capacitado ao exercício de atividades referentes à saúde bucal da população, pautado em princípios éticos, legais e na compreensão da realidade social, cultural e econômica do seu meio, dirigindo sua atuação para a transformação da realidade em benefício da sociedade."

No processo de territorialização, os discentes realizaram com êxito visitas domiciliares junto aos agentes comunitários de saúde (ACS). Estes profissionais atuam no território por meio de visitas domiciliares, ações de educação e orientações sobre cuidados com a saúde e prevenção das doenças estabelecendo uma peça fundamental na consolidação da Estratégia De Saúde da Família (ESF). O trabalho do ACS, desta forma, mantém-se como "ponte" entre os serviços de saúde e comunidade, fortalecendo o vínculo entre estes ${ }^{11}$.

A visita domiciliar é uma estratégia dentre outras técnicas, procedimentos e saberes da Saúde Coletiva, utilizado para a intervenção no processo saúde-doença familiar realizada no local de moradia, e não de trabalho ou estudo. Através das visitas é possível subsidiar a intervenção em problemas de saúde de indivíduos ou o planejamento de ações visando a promoção da saúde da coletividade. É possível também que a equipe de saúde conheça o contexto de vida do usuário, identifique as relações familiares, 
planeje melhor a assistência por permitir o reconhecimento dos recursos que a família dispõe e estabeleça vínculo entre estes ${ }^{12,13,14}$.

A exposição do estudante ao contexto de visitas domiciliares é de extrema importância para formação em odontologia, uma vez que a Política Nacional de Atenção Básica ${ }^{15}$ e a Política Nacional de Saúde Bucal ${ }^{16}$ recomenda que a atenção domiciliar seja realizada por equipes de saúde, incluindo a Equipe de Saúde Bucal (EqSB), que assistem indivíduos e famílias que tenham dificuldades para acessar os serviços de saúde de forma a promover o acesso às políticas públicas e representa um dos instrumentos que garantem a integralidade e equidade da atenção, princípios doutrinários do SUS.

No que tange as atividades educativas realizadas com a comunidade assistida, houve diversidade no público alvo, visto que as ações foram feitas para grupos específicos, como idosos, gestantes, mulheres, homens, indivíduos com doenças sistêmicas crianças e adolescentes. Todas as atividades foram realizadas utilizando metodologias ativas e métodos dialógicos que permitiram ao educando uma reflexão, pois como prediz Freire $(1987)^{17}$ :

“A concepção de educação como um processo que envolve açãoreflexão-ação, capacita as pessoas a aprenderem, evidenciando a necessidade de uma ação concreta, cultural, política e social visando "situações limites" e "superação das contradições."

Neste sentido, a educação em saúde é considerada uma ferramenta que permite o profissional aprofundar na realidade do usuário e após entender o contexto em que os indivíduos estão inseridos estimular a autonomia sobre o cuidado com a saúde individual, familiar e coletiva ${ }^{18}$.

Desta forma, existe uma relação entre educação em saúde e a pedagogia libertadora de Paulo Freire, que parte de um diálogo horizontalizado entre profissionais e usuários, contribui para a construção da emancipação do sujeito para o desenvolvimento da saúde individual e coletiva ${ }^{19}$.
As atividades de educação em saúde envolvendo o ambiente escolar foram consideradas essenciais, tendo em vista que o referido local é estratégico e de referência para o processo de desenvolvimento saudável, já que a escola é um lugar de formação e de inclusão social, transmissão de conhecimento e de desenvolvimento de hábitos promotores da saúde física e emocional, neste sentido, o público de idade escolar é um público alvo para prevenir doenças e promover saúde no âmbito da Estratégia de Saúde da Família ${ }^{19,20}$.

Durante as atividades educativas realizadas em ambiente escolar, foram realizadas também, como método de prevenção às doenças bucais, a escovação supervisionada e distribuição de kits de higiene bucal. A escovação supervisionada tem o objetivo de orientar e estimular os educandos a incorporar hábitos de higiene bucal, além de disponibilizar o flúor na cavidade bucal, por meio do creme dental, prevenindo, desta

forma, doenças como cárie e doença periodontal ${ }^{21,22}$.

A fim de conhecer a condição de saúde bucal foram realizados levantamentos epidemiológicos, considerando o índice CPO-d e ceo-d, número de dentes cariados, perdidos e obturados, além da necessidade de tratamento ${ }^{10}$. Para a Organização Mundial de Saúde $(\mathrm{OMS})^{23}$, os levantamentos fornecem um quadro de informações mais apuradas das condições de saúde bucal e das necessidades de tratamento de uma população, bem como podem proporcionar condições para controlar as mudanças nos níveis ou padrões da doença, além disso, os dados coletados em um levantamento epidemiológico podem ser utilizados para comparações em um momento futuro ${ }^{24}$.

Além de atividades educativas para a comunidade, as capacitações para profissionais das equipes de saúde também tiveram destaque. A capacitação sobre saúde bucal para as equipes de saúde das USFs foi de grande valia, buscou-se aprimorar os conhecimentos destes profissionais acerca de saúde bucal, doenças bucais mais prevalentes, medidas preventivas e de detecção precoce do câncer de boca, visto que o papel social destes é de grande relevância no contexto de mudanças das práticas de saúde junto à população. Por isso, a Educação Permanente em 
Saúde é fortemente valorizada na Atenção Primária, afinal, esta é um processo educativo que coloca o cotidiano do trabalho em saúde em análise, possibilita construir espaços coletivos para a reflexão e avaliação de sentido dos atos produzidos no cotidiano, ponderando sempre a realidade vivenciada ${ }^{25}$. Nesse sentido, a qualificação desses atores torna-se fundamental para ter alcance à comunidade em geral.

O estudante ao sair da sala de aula e vivenciar cotidianamente a realidade do sistema de saúde faz uma transição essencial para uma formação sólida e fundamentada na dinâmica do SUS, uma vez que tem a oportunidade única de ouvir dos que vivenciam diariamente o SUS, através de conversas com os usuários do serviço e profissionais que ali atuam, a partir desta experiência, o estudante de graduação tem um contato com os desafios e necessidades, criando assim uma consciência que os trabalhadores e usuários do SUS devem ser muito mais do que integrantes, estes devem também participar ativamente do processo de consolidação e defesa do direito à saúde.

A fim de realizar uma devolutiva do estágio para as unidades de saúde e para a população assistida, os dados coletados durante o estágio foram tabulados, analisados e apresentados às respectivas USFs. Durante a devolutiva foram discutidos juntamente com a equipe de enfermeiros coordenadores, cirurgião-dentista das unidades que possuíam este profissional, e agentes comunitários de saúde, as possíveis estratégias para o avanço de uma melhor assistência à saúde bucal das famílias assistidas. Além disso, os relatórios finais de cada unidade foram encaminhados e apresentados a gestão municipal de saúde.

Tal troca foi essencial também para a possibilidade de criação de uma visão crítica e fundamentada, capaz de absorver as opiniões de cada classe profissional sobre a realidade de saúde de cada localidade. Os estagiários expuseram à equipe, além dos dados, as suas vivências, experiências e visões acerca da área de abrangência de atuação, além de explorarem os pontos positivos, negativos e também desafios que foram observados durante a vivência, abrindo assim uma discussão sobre as possíveis propostas viáveis para o fortalecimento do sistema de saúde naquela área.
Por fim, faz-se necessário destacar que o docente de ensino superior da área de saúde coletiva deve atuar como mediador do processo de aprendizagem durante o estágio, empregando métodos e práticas pedagógicas pertinentes, norteando questões, trazendo motivação frente aos desafios encontrados, promovendo articulação entre teoria e prática através de metodologias ativas de aprendizagem, tendo o diálogo como eixo do processo educativo de forma a horizontalizar as relações e construir autonomia e co-responsabilização, tornando os estagiários atores do processo de ensinoaprendizagem e influenciando no futuro processo de trabalho do cirurgião-dentista em formação.

\section{Conclusão}

As disciplinas de cunho social nos cursos de saúde proporcionam ao discente uma abordagem mais crítica e reflexiva do processo saúde-doença. O estágio em saúde coletiva, por sua vez, possibilitou a participação acadêmica no desenvolvimento de ações de educação em saúde, as quais beneficiaram a comunidade assistida e a equipe da USF envolvida, uma vez que foram aplicados conhecimentos teóricopráticos a respeito da saúde bucal e geral,

levando informações muitas vezes desconhecidas. Ademais, o referido estágio permitiu ao discente à vivência da realidade do SUS, podendo promover uma geração de estudantes sensibilizados a uma prática social inerente e fundamental para trabalho neste sistema, visto que, os conhecimentos adquiridos e as práticas vivenciadas proporcionam ao estudante uma reflexão acerca do funcionamento, dinâmica, estratégias e desafios do SUS, o que fomenta o compromisso do estagiário perante seu papel como cidadão, estudante e futuro profissional de saúde e o mesmo descubra, assim, como deve comporta-se para integrar e cooperar fielmente com as necessidades do SUS.

\section{Referências}

1. Watanabe, MGC. Mudanças curriculares no curso de Odontologia da Faculdadede Odontologia de Ribeirão Preto da Universidade 
de São Paulo: um olhar para a aproximação com os serviços de saúde do Sistema Único de Saúde. Ribeirão Preto/SP. Tese [Livre-docência] Faculdade de Odontologia de Ribeirão Preto/Universidade de São Paulo; 2007.

2. Namen FM, Jorge RR, Galan Júnior J, Cabreira RD. Análise da Reforma Curricular em Odontologia no Estado do Rio de Janeiro. Rev. Bras. Ciên. Saúde 2008; 12(1): 55-80.

3. Conselho Nacional de Educação (Brasil). Resolução CNE/CES no 3, de 19 de fevereiro de 2002. Diretrizes Curriculares Nacionais do Curso de Graduação em Odontologia. Diário Oficial da União 04 de março de 2002; Seção 1.

4. Pereira, AC. Odontologia em Saúde Coletiva: planejando ações e promovendo saúde. Porto Alegre: Artmed; 2003.

5. Moysés, SJ. Políticas de saúde e formação de recursos humanos em Odontologia. Ver. ABENO 2001; 4(1):30-7.

6. Gomes de Pinho, MC. Trabalho em equipe de saúde: limites e possibilidades de atuação eficaz. Ciências \& cognição 2006; 8: 68-

87.

7. Ferreira, RC, Varga, CRR, Silva, RF. Trabalho em equipe multiprofissional: a perspectiva dos residentes médicos em saúde da família. CienSaudeColet 2009; 14: 1421-1428.

8. Instituto Brasileiro de Geografia e Estatística. Cidades [acesso: 25 de maio de 2018].

\section{Disponível}

https://cidades.ibge.gov.br/brasil/ba/feira-desantana/panorama.

9. Ministério da Saúde. Secretaria de Atenção à Saúde. Secretaria de Vigilância em Saúde. SB Brasil 2010: Pesquisa Nacional de Saúde Bucal: resultados principais. Brasília, DF: Ministério da Saúde, 2010.

10. Ministério da Saúde. Secretaria de Atenção à Saúde. Secretaria de Vigilância em Saúde. Projeto SB Brasil 2003: Condições de saúde bucal da população brasileira 2002-2003: resultados principais. Brasília, DF: Ministério da Saúde, 2003.

11. Fraga, LS, Monteiro, S. A gente é um passador de informação: práticas educativas de agentes de combate a endemias no serviço de controle de zoonoses em Belo Horizonte, MG. Saúde e Soc 2014; 23: 993-1006.

12. Egry,EY, Da Fonseca, RMGS. A família, a visita domiciliária e a enfermagem: revisitando o processo de trabalho da enfermagem em saúde coletiva. Rev. Esc. Enferm.USP 2000; 34(3): 233239.

13. Takahashi, RF; Oliveira, MAC. A visita domiciliária no contexto da Saúde da Família. In: Ministério da Saúde. (Org.). Manual de Enfermagem.São Paulo: Universidade de São Paulo, p. 43-46, 2001.

14. Lacerda, MR, Giacomozzi, CM, Oliniski, SR, Truppel, TC. Atenção à saúde no domicílio: modalidades que fundamentam sua prática. Saúde e Soc 2006; 15: 88-95.

15. Ministério da Saúde. Secretaria de Atenção à Saúde. Departamento de Atenção Básica. Política Nacional de Atenção Básica. Brasília, DF: Ministério da Saúde, 2012.

16. Ministério da Saúde. Secretaria de Atenção à Saúde. Departamento de Atenção Básica. Coordenação Nacional de Saúde Bucal. Diretrizes da Política Nacional de Saúde Bucal. Brasília, DF: Ministério da Saúde, 2004

17. Freire, P. Pedagogia do oprimido. 17a. Ed. Rio de Janeiro: Paz e Terra, 1987.

18. Freire P. Pedagogia da autonomia: saberes necessários à prática educativa. 34a ed. São Paulo: Paz e Terra, 1996.

19. Falkenberg, MB, Mendes, TPL, Moraes, EP, Souza, EM. Educação em saúde e educação na saúde: conceitos e implicações para a saúde coletiva. CienSaudeColet 2014;19(3):847-852.

20. Salci, MA, Maceno, P, Rozza, SG, Silva, DMGV, Boehs, AE, Heidemann, ITSB. Educação em saúde e suas perspectivas teóricas: algumas reflexões. Texto Contexto Enferm 2013;22(1):224-230.

21. Ministério da Saúde. Secretaria de Atenção à Saúde. Departamento de Atenção Básica. Guia de recomendações para o uso de fluoretos no Brasil. Brasília, DF: Ministério da Saúde, 2009.

22. Saito, MI. Adolescência, cultura, vulnerabilidade e risco. Pediatria (Säo Paulo) 2000, 22(3): 217-219.

23. Organização Mundial da Saúde. Levantamento epidemiológico básico de saúde bucal. 4. Ed. São Paulo: Santos, 1999.

24. Pereira, AC. Tratado de saúde coletiva em odontologia. Ed. Napoleão, 2009.

25. Acioli, S. Novas práticas em saúde: estratégias e táticas de grupos populares no enfrentamento de questões cotidianas. Estudos Saúde Coletiva 2000, 202(1): 3-17. 


\section{Endereço para Correspondência}

Universidade Federal de Campina Grande- UFCG

R. Aprígio Veloso, 882 - Universitário, Campina Grande - PB

CEP.:58428-830

e-mail: amorim.mah@hotmail.com

Recebido em 22/11/2019

Aprovado em 13/04/2020

Publicado em 30/03/2020 